KNEBUSCH, M.

Math. Zeitschr. 89, 213-223 (1965)

\title{
Assoziierte Vektoren in maximalen Gittern lokaler quadratischer Räume
}

\author{
Von \\ MANFRED KNEBUSCH
}

\section{Einleitung}

$k$ sei kompletter diskret bewerteter Körper einer Charakteristik $\neq 2$, $A$ der Ring der ganzen Elemente von $k$. Wir betrachten über $k$ einen Vektorraum $V$, versehen mit einer nicht ausgearteten quadratischen Form $F$, und in $V$ ein $A$-Gitter $M .(x, y)$ sei die Bilinearform $F(x+y)-F(x)-F(y)$. Für einen Vektor $e$ aus $V$ bezeichnen wir als ,Länge" den Wert $F(e)$, als ,Skala“ (bezüglich $M$ ) das $A$-Ideal $(e, M)$ in $k$.

Zwei Vektoren $e, h$ aus $M$ heißen assoziiert, wenn $e$ in $h$ durch eine Einheit von $M$, d.h. durch eine $M$ stabil lassende Isometrie von $V$, überführt werden kann. Wie kann man entscheiden, ob zwei Vektoren aus $M$ assoziiert sind oder nicht?

Das folgende, jedenfalls notwendige Kriterium bietet sich an:

(A) $e, h$ besitzen gleich Länge und Skala.

Wir werden in $\S 1$ dieser Arbeit beweisen, daß (A) auch hinreichend ist, falls $M$ maximal ist, d. h. sich nicht vergrößern läßt, ohne daß das von den Werten von $F$ auf $M$ erzeugte $A$-Ideal sich ändert.

Satz 1. In einem maximalen Gitter $M$ eines nicht ausgearteten quadratischen Raumes über diskret bewertetem (komplettem) Grundkörper sind Vektoren gleicher Länge und Skala assoziiert ${ }^{1}$ ).

Bei nicht maximalen Gittern oder allgemeineren Grundkörpern reichen Länge und Skala zur Festlegung einer Klasse assoziierter Vektoren gewöhnlich nicht aus. Das demonstrieren wir in $\S 2$ an Beispielen.

Zum Beweis von Satz 1 benötigen wir die Sätze 9.4 und 9.5 über maximale Gitter aus dem Buch [2] von EICHLER. Die in [2] generell vorausgesetzte Endlichkeit des Restklassenkörpers von $k$ wird bei diesen Sätzen nicht gebraucht.

Für beliebige Gitter $M$ scheint - schon bei den hier betrachteten Grundkörpern - die Angabe eines notwendigen und hinreichenden praktikablen Kriteriums dafür, daß zwei Vektoren aus $M$ assoziiert sind, zumindest schwierig zu sein. EICHLER gibt in [2], Satz 10.4 ein tiefliegendes hinreichendes Kriterium (B) an. Wir zeigen in §3, daß sich aus (B) stets (A) folgern läßt. Für den Spezialfall maximaler Gitter läßt sich das Eichlersche Resultat aufgrund von Satz 1 verschärfen (s. §3).

1) Siehe Bemerkung am Schluß der Einleitung. 
Satz 1 liefert schließlich, wie aus [3], §4 hervorgeht, den Schlüssel zur Klassifikation der Maximalordnungen einer einfachen lokalen Jordanalgebra vom Grade 2. Diese Klassifikation wird in $\$ 4$ der vorliegenden Arbeit explizit durchgeführt. Außerdem werden die Maximalordnungen bestimmt, die ein vollständiges Orthogonalsystem primitiver Idempotente enthalten.

Abschließend sei bemerkt, daß sich in den Sätzen 1 bis 4 dieser Arbeit die Voraussetzung, daß $k$ komplett ist, streichen läßt. Es gilt nämlich bei nicht komplettem $k$ : Läßt sich $e \in M$ in $h \in M$ durch eine Einheit $\bar{\sigma}$ des komplettierten Gitters $\bar{M}$ überführen, so auch durch eine Einheit von $M^{2}$ ). Zum Beweise approximiere man $\bar{\sigma}$ durch eine Einheit $\sigma$ von $M$ (vgl. [4], S. 291). Liegt $e^{\prime}:=\sigma(e)$ genügend nahe an $h$, so läßt sich $e^{\prime}$ in $h$ durch eine Einheit von $M$ überführen, etwa durch ein Produkt $S_{r} S_{t}$ benachbarter Spiegelungen.

$$
\left(S_{t}(x):=x-F(t)^{-1}(x, t) t .\right.
$$

Man suche ein $t \in M$ mit $F(t) \mid(t, M),(h, t) \neq 0$ und nehme

$$
r:=S_{t}\left(e^{\prime}\right)-h=\left(e^{\prime}-h\right)-F(t)^{-1}(h, t) t .
$$

Liegt der erste Vektor $e^{\prime}-h$ in dieser Differenz erheblich näher an Null als der zweite, so ist auch $S_{r}$ eine Einheit von $M$.)

\section{Weitere Bezeichnungen}

OE: „Ohne Einschränkung der Allgemeinheit“.

$R \subset S$ : Die Menge $R$ ist in der Menge $S$ enthalten, eventuell ist $R=S$.

$k^{*}$ : multiplikative Gruppe von $k$,

$\mathfrak{p}: \quad$ das Primideal von $A$,

$p$ : fest gewähltes Primelement von $A$,

$\left[p^{v}\right]: \quad$ nicht näher bestimmtes Element der Ordnung $v$ aus $k$.

$\alpha \sim \beta: \alpha=\beta \varepsilon$ mit einer Einheit $\varepsilon(\alpha, \beta \in k)$,

$\alpha \cong \beta: \alpha=\beta \varepsilon^{2}$ mit einer Einheit $\varepsilon(\alpha, \beta \in k)$,

$V_{0}$ : ein Kernraum von V.

Das Zeichen $\oplus$ bedeute nicht nur Direktheit im modultheoretischen Sinne, sondern zugleich Orthogonalität unter $F$. Ein ungeordnetes Paar $\left\{f_{1}, f_{2}\right\}$ von Vektoren aus $V$ heiße hyperbolisch, wenn $F\left(f_{1}\right)=F\left(f_{2}\right)=0,\left(f_{1}, f_{2}\right)=1$ ist.

\section{§1. Beweis von Satz 1}

1. Wir können $\mathrm{OE}$ voraussetzen, daß das maximale Gitter $M$ die Norm $A$ besitzt, da wir zu einer ähnlichen Form übergehen dürfen. Ist $V$ anisotrop, so ist Satz 1 trivial: Nach [2], Satz 10.1 ist jede Isometrie von $V$ Einheit von $M$. Zwei beliebige Vektoren gleicher Länge von $M$ sind aufgrund des Satzes von WITT (s. [7]) assoziiert.

2) Auf diesen Sachverhalt hat mich Herr Prof. M. KNESER hingewiesen. 
Sei also $V$ isotrop. Dann läßt sich von $M$ mindestens ein Gitter $A f_{1}+A f_{2}$ mit hyperbolischem Vektorpaar $f_{1}, f_{2}$ als direkter Summand abspalten (s. [2]; [4], 82:20, 82:21 a):

$$
M=N \oplus\left(A f_{1}+A f_{2}\right) .
$$

Als Vorbereitung zum Beweis von Theorem 1 vermerken wir, daß zu beliebig vorgegebenem $g \in N$ die Transformationen

$$
E_{\mathrm{g}}^{(i)}: x \rightarrow x+\left(x, f_{i}\right) g-(x, g) f_{i}-F(g)\left(x, f_{i}\right) f_{i}
$$

$\left(i=1,2 ; x \in V\right.$ ) Einheiten von $M$ sind ([6]; [2] S. 13, S. 58). $E_{g}^{(i)}$ führt $e=w+$ $\alpha_{1} f_{1}+\alpha_{2} f_{2}\left(w \in N, \alpha_{1}, \alpha_{2} \in A\right)$ in $e^{\prime}=w^{\prime}+\alpha_{1}^{\prime} f_{1}+\alpha_{2}^{\prime} f_{2}$ mit

$$
w^{\prime}=w+\alpha_{j} g, \quad \alpha_{i}^{\prime}=\alpha_{i}-(w, g)-\alpha_{j} F(g), \quad \alpha_{j}^{\prime}=\alpha_{j}
$$

über $((i, j)=(1,2)$ oder $(2,1))$.

$p r_{W}$ bezeichne die senkrechte Projektion von $V$ auf $W:=k N$ $E_{g}^{(i)}$ ist gekennzeichnet als die einzige Isometrie $\sigma$ von $V$, die $f_{i}$ festläßt und für die $p r_{W} \circ \sigma$ den Vektor $f_{j}$ in $g$ überführt und auf $W$ die Identität ist.

2. Das folgende Lemma 1.1 zeigt, daß wir für den Beweis von Satz $1 \mathrm{OE}$ $\operatorname{dim} V-\operatorname{dim} V_{0}=2$ voraussetzen können.

Lemma 1.1. $M$ sei Gitter der Form $M_{0} \oplus\left(A f_{1}+A f_{2}\right) \oplus \cdots \oplus\left(A f_{2 t-1}+\right.$ $\left.A f_{2 t}\right)$ mit hyperbolischen Vektorpaaren $\left\{f_{2 v-1}, f_{2 v}\right\}(v=1, \ldots, t)$.

$$
e=e_{0}+\sum_{v=1}^{2 t} \alpha_{v} f_{v}
$$

sei Vektor aus $M$ mit ord $\alpha_{1}=\operatorname{Min}\left(\operatorname{ord} \alpha_{1}, \ldots\right.$, ord $\alpha_{2}$ ). Dann ist e in $M$ assoziiert zu einem Vektor $e_{0}+\alpha_{1} f_{1}+\alpha_{2}^{\prime} f_{2}$.

Beweis. Sei

$$
N:=M_{0} \oplus \sum_{v=3}^{2 t} A f_{v}, \quad g:=-\alpha_{1}^{-1} \sum_{v=3}^{2 t} \alpha_{v} f_{v} \in N .
$$

Wir bilden $E_{g}^{(2)}$ bezüglich der Zerlegung $M=N \oplus\left(A f_{1}+A f_{2}\right)$. Unter $E_{g}^{(2)}$ geht $e$ in $e_{0}+\alpha_{1} f_{1}+\left(\alpha_{2}+\alpha_{1} F(g)\right) f_{2}$ über (s. (1.3)).

3. Sei ab jetzt also $\operatorname{dim} V=\operatorname{dim} V_{0}+2, M$ maximales Gitter der Norm $A$ von $V$.

Lemma 1.2. $e, h^{\prime}$ seien Vektoren gleicher Länge des maximalen Gitters $M$.

Behauptung. Es gibt eine Witt-Zerlegung

$$
M=M_{0} \oplus\left(A f_{1}+A f_{2}\right)
$$

von $M$, einen $z u h^{\prime}$ assoziierten Vektor $h$ und einen Exponenten $r \geqq 0$, so daß

$$
e=e_{0}+\alpha_{1} f_{1}+\alpha_{2} f_{2}, \quad h=e_{0}+\beta_{1} f_{1}+\beta_{2} f_{2}
$$


mit gleichem $e_{0}$ und

$$
\beta_{1}=\alpha_{1} p^{r}, \quad \beta_{2}=\alpha_{2} p^{-r}
$$

ist.

Beweis. Wir gehen von irgendeiner, nach [7] sicher vorhandenen Isometrie $\sigma$ von $V$ aus, die $e$ in $h^{\prime}$ überführt. Aufgrund von [2], Satz 9.5 läßt sich eine Zerlegung (1.4) von $M$ finden, so daß

$$
\sigma^{-1}(M)=M_{0} \oplus\left(A p^{-r} f_{1}+A p^{r} f_{2}\right)
$$

wird mit geeigneter, OE positiver Primzahlpotenz $r$ (s. auch [4], 82:23).

Sei bezüglich dieser Wittzerlegung

$$
e=e_{0}+\alpha_{1} f_{1}+\alpha_{2} f_{2} \quad\left(e_{0} \in M_{0}, \alpha_{1}, \alpha_{2} \in A\right) .
$$

Anwendung von $\sigma$ auf (1.7) und (1.8) liefert:

$$
\begin{aligned}
& M=\sigma\left(M_{0}\right) \oplus\left[A p^{-r} \sigma\left(f_{1}\right)+A p^{r} \sigma\left(f_{2}\right)\right], \\
& h^{\prime}=\sigma\left(e_{0}\right)+\alpha_{1} \sigma\left(f_{1}\right)+\alpha_{2} \sigma\left(f_{2}\right) .
\end{aligned}
$$

Durch $x \rightarrow \sigma^{-1}(x)$ für $x \in \sigma\left(M_{0}\right), p^{-r} \sigma\left(f_{1}\right) \rightarrow f_{1}, p^{r} \sigma\left(f_{2}\right) \rightarrow f_{2}$ wird eine Einheit von $M$ definiert, die $h^{\prime}$ in $h:=e_{0}+\alpha_{1} p^{r} f_{1}+\alpha_{2} p^{-r} f_{2}$ überführt. q.e.d.

4. Seien jetzt $e, h$ Vektoren gleicher Länge $l$ und gleicher Skala $\mathfrak{p}^{v}$ unseres maximalen Gitters $M$. Aufgrund von Lemma 1.2 dürfen wir die durch (1.4) bis (1.6) beschriebene Situation voraussetzen.

Ist $r=0$, so sind wir fertig. Sei jetzt also $r>0$. Es ist

$$
\left(e_{0}, M_{0}\right)+\alpha_{1} A+\alpha_{2} A=\left(e_{0}, M_{0}\right)+\beta_{1} A+\beta_{2} A=\mathfrak{p}^{v},
$$

insbesondere also ord $\alpha_{1}$, ord $\alpha_{2}$, ord $\beta_{1}$, ord $\beta_{2} \geqq v$. Aus (1.6) ergibt sich für $\alpha_{2}, \beta_{1}$ genauer

$$
\text { ord } \alpha_{2}>v, \quad \text { ord } \beta_{1}>v \text {. }
$$

a) Sei $\left(e_{0}, M_{0}\right) \varsubsetneqq \mathfrak{p}^{v}$.

Man liest aus (1.9) und (1.10) ab: ord $\alpha_{1}=v$, ord $\beta_{2}=v$. Die durch

$$
f_{1} \rightarrow \beta_{2} \alpha_{1}^{-1} f_{2}, \quad f_{2} \rightarrow \alpha_{1} \beta_{2}^{-1} f_{1}, \quad x \rightarrow x, \quad \text { falls } x \in M_{0},
$$

definierte Einheit von $M$ führt $e$ in $h$ über. Es ist nämlich

$$
\alpha_{1} \alpha_{2}=l-F\left(e_{0}\right)=\beta_{1} \beta_{2} \text {, also } \alpha_{1} \beta_{2}^{-1}=\beta_{1} \alpha_{2}^{-1} \text {. }
$$

b) Sei $\left(e_{0}, M_{0}\right)=\mathfrak{p}^{v}$.

Wir zeigen: Es gibt ein $g \in M_{0}$ mit $\left(e_{0}, g\right)+\alpha_{1} F(g)=\alpha_{2}$. Zunächst läßt sich ein $h \in M_{0}$ finden mit $\left(e_{0}, h\right)=p^{v}$, also $w=\alpha_{2} p^{-v} h$ mit $\left(e_{0}, w\right)=\alpha_{2}$. Wir machen den Ansatz $g=\lambda w$ mit $\lambda \in A$ und haben dann zu lösen:

$$
\lambda \alpha_{2}+\lambda^{2} \alpha_{1} \alpha_{2}^{2} p^{-2 v} F(h)=\alpha_{2},
$$


also $c \lambda^{2}+\lambda-1=0$ mit $c:=\alpha_{1} \alpha_{2} p^{-2 v} F(h) \in \mathfrak{p}$ (s. (1.10)). Aufgrund des Henselschen Lemmas (s. z. B. [4], 13:9) läßt sich diese Gleichung mit $\lambda \in A$ erfüllen.

Mit einem so gefundenen $g$ bilden wir bezüglich unserer Witt-Zerlegung (1.4) die Einheit $E_{g}^{(2)}$ (s. (1.2)).

$E_{g}^{(2)}$ führt $e$ in den Vektor $e^{\prime}=b_{0}+\alpha_{1} f_{1}$ mit $b_{0}:=e_{0}+\alpha_{1} g$ über (s. (1.3)).

Es ist $\alpha_{1}\left(g, M_{0}\right)=\lambda \alpha_{1} \alpha_{2} p^{-v}\left(h, M_{0}\right) \subset \mathfrak{p}^{v+1}$ (s. (1.10)), also wieder $\left(b_{0}, M_{0}\right)$ $=\mathfrak{p}^{v}$. Deshalb existiert sicherlich $t \in M_{0}$ mit $\left(b_{0}, t\right)=\alpha_{1}$. $E_{t}^{(1)}$ führt $e^{\prime}$ in $b_{0}$ über (s. (1.3)).

Die durch (1.4) bis (1.6) und die Voraussetzung b) beschriebene Ausgangssituation ist in $e, h$ symmetrisch. Daher läßt sich auch $h$ durch eine Einheit von $M$ in einen Vektor $c_{0}$ von $M_{0}$ überführen. $b_{0}, c_{0}$ haben gleiche Länge, sind also assoziiert. q.e.d.

\section{§2. Beispiele}

1. Sei $k$ komplett diskret bewertet, $V$ der quadratische Raum

$k f_{0} \oplus\left(k f_{1}+k f_{2}\right) \quad$ mit $\quad F\left(f_{0}\right)=1, \quad F\left(f_{1}\right)=F\left(f_{2}\right)=0, \quad\left(f_{1}, f_{2}\right)=1$.

Wir betrachten in dem nicht maximalen Gitter $M:=\mathfrak{p} f_{0} \oplus\left(A f_{1}+A f_{2}\right)$ die Vektoren $e:=p\left(f_{0}+f_{1}\right), h:=p\left(f_{1}+f_{2}\right)$. $e, h$ haben beide die Länge $p^{2}$ und bezüglich $M$ die Skala $p$, sind aber nicht assoziiert, liegt doch $p^{-1} h$ in $M$, $p^{-1} e$ nicht in $M$.

2. Sei $k$ komplett diskret bewertet, $2 \in \mathfrak{p}$. Der Restklassenkörper von $k$ bestehe aus mehr als 2 Elementen. $M$ sei das Gitter der Form $A e_{1} \oplus A e_{2}$ mit $F\left(e_{1}\right)=-F\left(e_{2}\right)=1$.

Wir betrachten die Vektoren

$$
e=e_{1}-e_{2}, \quad h=\varepsilon\left(e_{1}-e_{2}\right)
$$

mit einer Einheit $\varepsilon$ von $A$, für die $\varepsilon^{2}+1 \notin 2 A$ ist. Eine solche Einheit gibt es wegen unserer Voraussetzung über den Restklassenkörper von $k$.

$e, h$ besitzen beide die Länge 0 und die Skala $2 A$. Es ist sogar

$$
\{x \in M \mid(x, e) \in \mathfrak{a}\}=\{x \in M \mid(x, h) \in \mathfrak{a}\}
$$

für jedes Ideal a von $A$. Wir zeigen, da $e, h$ trotzdem nicht assoziiert sind:

Man rechnet leicht nach, daß die Einheiten von $M$ die Transformationen $e_{1} \rightarrow \lambda e_{1}+\mu e_{2}, e_{2} \rightarrow \pm\left(\mu e_{1}+\lambda e_{2}\right)$ mit $\lambda, \mu \in A, \lambda^{2}-\mu^{2}=1$ sind. Sie führen $e$ in $(\lambda-\mu) e$ bzw. $(\lambda+\mu)\left(e_{1}+e_{2}\right)$ über. Also gilt: $e$ zu $h$ assoziiert $\Leftrightarrow \lambda^{2}-(\lambda-\varepsilon)^{2}=1$ mit $\lambda \in A$ lösbar $\Leftrightarrow \varepsilon^{2}+1 \in 2 A$, und das sollte nicht der Fall sein.

3. $k$ sei Quotientenkörper eines Dedekindringes $A$, der mindestens zwei Primideale besitzt. Es gibt in $A$ dann teilerfremde Nichteinheiten $n_{1}, n_{2}$. Wir betrachten die hyperbolische Ebene $V=k f_{1}+k f_{2}\left(F\left(f_{1}\right)=F\left(f_{2}\right)=0,\left(f_{1}, f_{2}\right)\right.$ =1) und darin das maximale Gitter $M:=A f_{1}+A f_{2} . e:=n_{1} f_{1}+n_{2} f_{2}$ und $h:=n_{1} n_{2} f_{1}+f_{2}$ haben beide die Länge $n_{1} n_{2}$ und die Skala $A$ bezüglich $M$. 
Die Einheiten von $M$ sind genau die Transformationen $f_{1} \rightarrow \varepsilon f_{1}, f_{2} \rightarrow \varepsilon^{-1} f_{2}$ und $f_{1} \rightarrow \eta f_{2}, f_{2} \rightarrow \eta^{-1} f_{1}$ mit Einheiten $\varepsilon, \eta$ von $A$. Man sieht unmittelbar, daß $e$ nicht $\mathrm{zu} h$ assoziiert ist.

\section{§3. Zusammenhang mit einem Kriterium von Eichler}

$k$ sei diskret bewerteter Körper einer Charakteristik $\neq 2$. $M$ sei beliebiges $A$-Gitter eines nichtausgearteten quadratischen Raumes $V$ über $k$ und besitze die Norm $n(M)=a$. Als Komplement von $M$ bezeichnet EICHLER in [2] das Gitter $\widetilde{M}:=\{x \in V \mid(x, M) \subset \mathfrak{a}\}$, als Stufe von $M$ das ganze $A$-Ideal $\mathfrak{q}:=n(M)$. $n(\tilde{M})^{-1}$, als Differente von $M$ das Gitter $D(M):=\mathfrak{q} M$. Man überzeugt sich leicht, daß $D(M)$ in $M$ enthalten ist. Komplement, Stufe und Differente ändern sich nicht, wenn man von der vorgegebenen Form zu einer ähnlichen übergeht.

EICHLER betrachtet in [2], S.60ff., Vektoren $e, h \in M$ mit der Eigenschaft (B) $e, h$ sind primitiv in $M$ und von gleicher Länge. $e-h$ liegt in $D(M)$. Er beweist darüber folgenden

Satz 2. $M$ sei beliebiges Gitter eines nichtausgearteten quadratischen Raumes über diskret bewertetem, komplettem Grundkörper $k$. Behauptung: Zwei der Bedingung (B) genügende Vektoren e, h von M sind assoziiert ${ }^{1}$ ) (s. [2], Satz 10.4).

Bemerkung. Die in [2] außerdem gemachte Voraussetzung, daß $k$ endlichen Restklassenkörper besitzt, läßt sich durch leichte Abänderung des Eichlerschen Beweisgedankens eliminieren.

Wir fragen, ob aus (B) die in der Einleitung formulierte Aussage (A) folgt. Das ist in der Tat der Fall. Es gilt sogar

Satz 3. $M$ sei beliebiges Gitter eines nichtausgearteten quadratischen Raumes über einem diskret bewerteten Körper $k . C(M)$ bezeichne das größte in $M$ enthaltene Gitter der Form $\mathfrak{b} \widetilde{M},(\tilde{M}=$ Komplement von $M, \mathfrak{b}$ Ideal von A). e, $h$ seien primitive Vektoren von $M$, deren Differenz in $C(M)$ liege.

Behauptung. $(e, M)=(h, M)$.

Bemerkung. Man rechnet sofort nach, daß $C(M)=\mathfrak{c} \tilde{M}$ mit $\mathfrak{c}:=n(M)$. $(\tilde{M}, \widetilde{M})^{-1}$ ist. Ist $\mathfrak{p}$ gerade, so kann $C(M) \nsupseteq D(M)$ sein. Sei z. B. $M$ modular, d.h. jeder primitive Vektor von $M$ besitze die Skala $(M, M)$. Die Norm von $M$ sei der Einfachheit halber $A$. Dann ist $\widetilde{M}=(M, M)^{-1} M, C(M)=M$, $D(M)=(M, M) M$. Für $(M, M)$ kommen alle Ideale zwischen $A$ und $2 A$ vor.

Beweis von Satz 3. $M$ habe $\mathrm{OE}$ die Norm $A$. Wir können $M$ in modulare Gitter $M_{i}$ zerlegen: $M=M_{1} \oplus \cdots \oplus M_{t}$. (Siehe [4], S. 243; die in [4] gemachten Voraussetzungen, da $B k$ komplett ist und endlichen Restklassenkörper besitzt, werden an dieser Stelle nicht gebraucht.) Für die Ideale $\mathfrak{s}_{i}:=\left(M_{i}, M_{i}\right)$ dürfen wir $\mathrm{OE}$ annehmen, daß $\mathfrak{s}_{i+1} \mathfrak{s}_{i}(i=1, \ldots, t-1)$ ist. Man berechnet: $\widetilde{M}=$ $\mathfrak{s}_{1}^{-1} M_{1} \oplus \cdots \oplus \mathfrak{s}_{t}^{-1} M_{t}, \mathfrak{c}=(\widetilde{M}, \widetilde{M})^{-1}=\mathfrak{s}_{t}$, schließlich

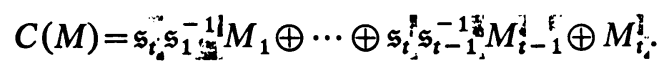


Nach Voraussetzung ist $(e-h, M) \subset \mathrm{c}$. Ist $(e, M) \supsetneq \mathrm{c}$, so ist daher sicherlich $(e, M)=(h, M)$.

Sei jetzt $(e, M) \subset \mathfrak{c},(h, M) \subset \mathfrak{c}$, d.h. $e \in C(M), h \in C(M)$. Wir schreiben: $e=e_{1}+\cdots+e_{t}, h=h_{1}+\cdots+h_{t}\left(e_{i}, h_{i} \in M_{i}\right), e, h$ sollten primitive Vektoren von $M$ sein. Ein Blick auf (3.1) lehrt: $e_{t}, h_{t}$ sind primitive Vektoren von $M_{t}$. Somit ist $(e, M) \supset\left(e_{t}, M_{t}\right)=\mathfrak{s}_{t}=\mathfrak{c},(h, M) \supset \mathfrak{c}$, also $(e, M)=(h, M)=\mathfrak{c}, \quad$ q.e.d.

Aus Satz 1 und 3 ergibt sich sofort eine Verschärfung des Eichlerschen Satzes im Spezialfall maximaler Gitter.

Satz 4. $M$ sei maximales Gitter eines nichtausgearteten quadratischen Raumes über diskret bewertetem (komplettem) Körper. $e$, h seien primitive Vektoren gleicher Länge in $M$, deren Differenz in $C(M)$ liegt. Dann sind e und h assoziiert ${ }^{1}$ ).

Die Frage, ob sich Satz 4 auf beliebige Gitter ausdehnen läßt, muß bei dyadischem Grundkörper verneint werden. Man betrachte als Gegenbeispiel wieder das in $\S 2.2$ definierte modulare Gitter $M$ und die primitiven isotropen Vektoren (2.1).

\section{§4. Klassifikation der Maximalordnungen einer einfachen lokalen Jordanalgebra vom Grade 2}

Sei $\mathfrak{Q}$ einfache Jordanalgebra vom Grade 2 über einem diskret bewerteten kompletten Körper $k$. $\mathfrak{A}$ ist unter der Minimalnorm $M N$ quadratischer Raum. Wir erfassen so alle nicht ausgearteten quadratischen Räume, die Vektoren der Länge 1 enthalten (s. [1]; [3], §4). $(x, y)$ sei die Bilinearform $M N(x+y)$ $M N(x)-M N(y)$. Die Maximalanordnungen von $\mathfrak{A}$ sind die maximalen Gitter der Norm $A$, die das Einselement $e$ enthalten (s. [3], § 4). Wir setzen grundsätzlich voraus, daß $M N$ isotrop, also $\mathfrak{A}$ reduziert ist. Anderenfalls gäbe es in $\mathfrak{A}$ überhaupt nur eine Maximalordnung (s. [2], Satz 9.4; [3], § 3).

$M$ bezeichne jetzt stets ein fest ausgewähltes maximales Gitter der Norm $A$ in $\mathfrak{A}$. $\mathrm{Zu}$ zwei Maximalanordnungen $B_{i}(i=1,2)$ gibt es Isometrien $\varphi_{i}$, die $B_{i}$ in $M$ überführen ([2], Satz 9.6).

Nun gilt nach [3], Satz 4.5:

(4.1) $B_{1}$ isomorph zu $B_{2} \Leftrightarrow \varphi_{1}(e), \varphi_{2}(e)$ in $M$ assoziiert $^{3}$ ).

Beachtet man noch, daß $\left(\varphi_{i}(e), M\right)=\left(e, B_{i}\right)$ ist, so liefert Satz 1 sofort

Satz 1.a. Zwei Maximalanordnungen $B_{1}, B_{2}$ einer einfachen Jordanalgebra vom Grade 2 mit Einselement e über diskret bewertetem (komplettem) Körper $k$ sind genau dann isomorph, wenn $\left(e, B_{1}\right)=\left(e, B_{2}\right)$ ist $\left.{ }^{3}\right)$.

Wir fragen, für welche Werte $(e, B)$ eine Maximalordnung $B$ ein Idempotent $u \neq e$, also ein vollständiges Orthogonalsystem $u, e-u$ absolut primitiver Idempotente enthält.

Satz 5. $k$ sei diskret bewertet. $\mathfrak{A}$ sei einfache reduzierte Jordanalgebra vom Grade 2 über $k$ mit Einselement $e$.

3) In [2], Satz 9.6 und daher auch hier läßt sich die Voraussetzung, daß $k$ komplett ist, durch ein Approximationsargument nachträglich eliminieren (vgl. Schluß der Einleitung). 
Behauptung. Eine Maximalordnung $B$ von $\mathfrak{A}$ enthält genau dann ein Idempotent $\neq e$, wenn $(e, B)=A$ ist.

Beweis. Ein Idempotent $u \neq e$ läßt sich charakterisieren als Element von $\mathfrak{Q}$, das genau die Eigenwerte 0 und 1 besitzt, also (s. z. B. [3], (1.19), (1.20), (4.2)) den Gleichungen $(u, e)=1, M N(u)=0$ genügt. Enthält $B$ ein solches Idempotent, so ist sicher $(e, B)=A$.

Sei umgekehrt $(e, B)=A$ vorausgesetzt. Weil $M N$ isotrop ist, besitzt $B$ eine orthogonale Zerlegung $B=B^{\prime} \oplus\left(A f_{1}+A f_{2}\right)$ mit hyperbolischem Vektorpaar $f_{1}, f_{2}$ (s. z. B. [4], 82:20, 82:21 a). Sei $e=e^{\prime}+\alpha_{1} f_{1}+\alpha_{2} f_{2}\left(e^{\prime} \in B^{\prime}, \alpha_{1}, \alpha_{2} \in A\right)$. Nach Voraussetzung existiert ein $x=x^{\prime}+\xi_{1} f_{1}+\xi_{2} f_{2}\left(x^{\prime} \in B^{\prime}, \xi_{1}, \xi_{2} \in A\right)$ mit

$$
(x, e)=\left(x^{\prime}, e^{\prime}\right)+\alpha_{1} \xi_{2}+\alpha_{2} \xi_{1}=1 \text {. }
$$

1. Fall: $\alpha_{1} \sim 1$.

$u=\alpha_{1}^{-1} f_{2}$ ist primitives Idempotent in $M$.

2. Fall: $\alpha_{1} \in \mathfrak{p}, \alpha_{2} \in \mathfrak{p}$.

Aus (4.2) folgt $\left(x^{\prime}, e^{\prime}\right) \sim 1$. Man wähle $\zeta_{1}, \zeta_{2}$ irgendwie in $A$ so, da $\zeta_{1} \zeta_{2}=$ $-M N\left(x^{\prime}\right)$ ist. Für $w:=x^{\prime}+\zeta_{1} f_{1}+\zeta_{2} f_{2}$ ist $M N(w)=0,(w, e)=\left(x^{\prime}, e^{\prime}\right)+$ $\alpha_{1} \zeta_{2}+\alpha_{2} \zeta_{1} \sim 1$.

$u:=(w, e)^{-1} w \in M$ ist Idempotent $\neq e . \quad$ q.e.d.

Satz 6. $k$ sei diskret bewertet, 2 sei Einheit. Dann sind alle Maximalordnungen $B$ von $\mathfrak{A}$ isomorph und enthalten Idempotente $\neq e$.

Beweis. Wegen $(e, e)=2 \sim 1$ ist $(e, B)=A$.

Satz 7. A sei beliebiger Dedekindring. $\mathfrak{A}$ habe keinen Kernraum. Dann enthält jede Maximalordnung von $\mathfrak{A}$ ein Idempotent $\neq e$. Ist A Hauptidealring, so sind alle Maximalordnungen von $\mathfrak{A}$ isomorph.

Beweis. Eine Maximalordnung $B$ besitzt eine Zerlegung

$$
B=\oplus \sum_{i=1}^{t}\left(\mathfrak{b}_{i} f_{2 i-1}+\mathfrak{b}_{i}^{-1} f_{2 i}\right)
$$

mit $A$-Idealen $\mathfrak{b}_{i}$ von $k$ und hyperbolischen Vektorpaaren $\left\{f_{2 i-1}, f_{2 i}\right\}$ (s. [4], $82: 20,82: 21$ a). Sei

Für

$$
e=\sum_{i=1}^{t}\left(\alpha_{2 i-1} f_{2 i-1}+\alpha_{2 i} f_{2 i}\right) .
$$

$$
\begin{aligned}
u & :=\sum_{i=1}^{t} \alpha_{2 i-1} f_{2 i-1} \in B \quad \text { gilt } M N(u)=0, \\
(u, e) & =\sum_{i=1}^{t} \alpha_{2 i-1} \alpha_{2 i}=M N(e)=1 .
\end{aligned}
$$

$u$ ist Idempotent $\neq e$ in $B$. $\mathrm{Zu}$ dem hyperbolischen Vektorpaar $u, v:=e-u$ gibt es eine Zerlegung

$$
B=(A u+A v) \oplus D \quad \text { (s. [4], 82:15). }
$$


Sei $B_{1}=\left(A u_{1}+A v_{1}\right) \oplus D_{1}$ eine weitere, ebenso zerlegte Maximalordnung. $D, D_{1}$ sind maximale Gitter gleicher Norm in isometrischen hyperbolischen Räumen. Ist $A$ Hauptidealring, so sind $D, D_{1}$ selbst isometrisch. Es gibt dann eine Isometrie $\sigma$ von $V$ auf sich mit $\sigma(D)=D_{1}, \sigma(u)=u_{1}, \sigma(v)=v_{1} . \sigma$ läßt $e=u+v=u_{1}+v_{1}$ fest, ist also ein Automorphismus der Algebra $\mathfrak{A}$ (s. z. B. [3], § 4) und führt $B$ in $B_{1}$ über. q.e.d.

Wir wollen uns abschließend für kompletten, diskret bewerteten Grundkörper $k$ mit endlichem Restklassenkörper auch, falls 2 nicht Einheit ist, eine Übersicht über die Klassen isomorpher Maximalordnungen $B$ von $\mathfrak{A}$ verschaffen, indem wir angeben, welche $\mathfrak{p}^{v}$ als Ideale $(e, B)$ vorkommen.

Sei $\mathfrak{A}_{0}$ Kernraum von $\mathfrak{A}, \Delta$ Element der Ordnung 0 oder 1 von $k$, welches die signierte Diskriminante von $\mathfrak{A}$ repräsentiert: $\Delta=(-1)^{s(s-1) / 2} \operatorname{det}\left(\frac{1}{2}\left(x_{i}, x_{j}\right)\right)$ für eine Basis $x_{1}, \ldots, x_{s}$ von $\mathfrak{A}$. Ist $\operatorname{dim} \mathfrak{H}_{0}=2$, so schreiben wir $\mathfrak{H}$ den Wert $\chi=+1 \mathrm{zu}$, falls $\mathfrak{I}_{0}$ einen Vektor der Länge 1 enthält, sonst den Wert $\chi=-1$. Mit $w$ bezeichnen wir eine festgewählte Einheit der Form $1+[4]$ von $A$, für die $k(\sqrt{w}) / k$ unverzweigt vom Grade 2 ist.

Satz 8. Il sei reduzierte einfache Jordanalgebra vom Grade 2 mit Einselement $e$ über einem diskret bewerteten, kompletten Körper $k$ mit endlichem Restklassenkörper. $\omega:=$ ord 2 sei $>0$.

Behauptung. Die Gesamtheit der Ideale $(e, B)=\mathfrak{p}^{v} z u$ den Maximalordnungen $B$ von $\mathfrak{A}$ hängt nur von dem Raumtyp von $\mathfrak{A}$ ab. Es treten folgende Exponenten $v$ auf:

$\operatorname{dim} \mathfrak{A}_{0}=0$ oder 4 :

$\operatorname{dim} \mathfrak{A}_{0}=1$ :

$$
\begin{aligned}
& \Delta \cong 1 \text { oder } w \\
& \Delta \cong 1+\left[p^{2 g+1}\right], g<\omega \\
& \Delta \sim p
\end{aligned}
$$$$
v=0 \text {; }
$$$$
0 \leqq v \leqq \omega
$$$$
0 \leqq v \leqq g
$$

$\Delta \cong w$$$
v=0 \text {; }
$$

$\operatorname{dim} \mathfrak{A}_{0}=2$ :

$$
\begin{array}{rl}
\Delta \cong 1+\left[p^{2 g+1}\right], g<\omega ; \chi=-1 & 0 \leqq \nu \leqq \omega-g-1 \\
& =+1 \quad 0 \leqq \nu \leqq \omega-g \\
\Delta \sim p & 0 \leqq \nu \leqq \omega ;
\end{array}
$$

$\operatorname{dim} \mathfrak{A}_{0}=3:$

$$
\begin{aligned}
& \Delta \cong 1 \text { oder } w \\
& \Delta \cong 1+\left[p^{2 g+1}\right], g<\omega \\
& \Delta \sim p
\end{aligned}
$$$$
0 \leqq v \leqq \omega
$$$$
0 \leqq v \leqq g+1
$$$$
v=0 \text {. }
$$

Beweis. Wegen $(e, e)=2 \in(e, B) \operatorname{mu} ß$ stets $0 \leqq v \leqq \omega$ sein. Die Gesamtheit der Ideale $(e, B)$ ist zugleich die Gesamtheit der Ideale $(h, M)$, wobei $h$ alle Vektoren der Länge $M N(e)=1$ unseres festgewählten maximalen Gitters $M$ durchlaufe (s. [3], Satz 4.5). Sie hängt nach Lemma 1.1 nur von dem Raumtyp von $\mathfrak{A}$ ab. Wir nehmen $\mathrm{OE}$ an, $\operatorname{da} \beta \operatorname{dim} \mathfrak{A}-\operatorname{dim} \mathfrak{A}_{0}=2$ ist. Sei $M=$ $M_{0} \oplus\left(A f_{1}+A f_{2}\right)$ eine festgewählte Wittzerlegung von $M$ mit hyperbolischem Vektorpaar $\left\{f_{1}, f_{2}\right\}$. Mit $\mathfrak{I}_{0}$ sei jetzt genauer der Kernraum $k M_{0}$ bezeichnet. 
Wir betrachten also für die Vektoren $h=h_{0}+\alpha_{1} f_{1}+\alpha_{2} f_{2}\left(h_{0} \in M_{0}, \alpha_{1}\right.$, $\left.\alpha_{2} \in A\right)$ mit

$$
M N\left(h_{0}\right)+\alpha_{1} \alpha_{2}=1
$$

und OE mit ord $\alpha_{1} \leqq$ ord $\alpha_{2}$ die Skalen

$$
\mathfrak{p}^{v}:=(h, M)=\left(h_{0}, M_{0}\right)+\alpha_{1} A+\alpha_{2} A \text {. }
$$

Hat $h$ die Skala $p^{v}$, so gibt es auch zu jedem $\mu$ mit $0 \leqq \mu<v$ Vektoren der Länge 1 und Skala $\mathfrak{p}^{\mu}$ in $M$, z. B. $h^{\prime}=h_{0}+p^{\mu} f_{1}+\alpha_{1} \alpha_{2} p^{-\mu} f_{2}$.

Insbesondere komme die Skala $A$ stets vor (Beispiel: $h=f_{1}+f_{2}$ ). Auf der Suche nach Vektoren $h$ einer Skala $\subset \mathfrak{p}$ können wir von vornherein annehmen, daß $\alpha_{1}$ und $\alpha_{2}$ nicht Einheiten sind (s. (4.5)). Nach (4.4) ist dann

$$
M N\left(h_{0}\right) \sim 1 .
$$

Wir gehen jetzt die verschiedenen Raumtypen durch. Wegen (4.6) (oder Satz 7) ist der Fall $\mathfrak{U}_{0}=0$ schon erledigt.

$\operatorname{dim} \mathfrak{A}_{0}=1$. Es ist $M_{0}=A a$ mit $M N(a)=\Delta$. (4.6) zeigt, daß der Fall $\Delta \sim p$ schon ausgeschaltet ist. Ist $\Delta=1$, so kommt $v=\omega$ vor. Beispiel: $h=a$. Dasselbe gilt, falls $\Delta=w=1+4 \varepsilon(\varepsilon \sim 1)$ ist. Beispiel: $h=a+2 f_{1}-2 \varepsilon f_{2}$.

Sei $\Delta=1+\varepsilon p^{2} g+1(g<\omega, \varepsilon \sim 1) . v=g+1$ kommt nicht als Exponent der Skala eines Vektors $h$ der betrachteten Art vor. Denn für den Skalar $\lambda$ mit $h_{0}=\lambda a$ würde aus (4.4) folgen: $\lambda^{2} \Delta \equiv 1 \bmod \mathfrak{p}^{2 g+2} . \Delta$ besitzt aber den quadratischen Defekt $\mathfrak{p}^{2 g+1}$ (s. z.B. [4], 63:5). $v=g$ kommt vor. Beispiel: $h=$ $a+p^{g} f_{1}-\varepsilon p^{g+1} f_{2}$.

$\operatorname{dim} \mathfrak{A}_{0}=2$. Wir identifizieren $\mathfrak{A}_{0}$ mit $K:=k(\sqrt{\Delta})$ unter einer quadratischen Form $M N(x)=\lambda N(x) . N$ bedeute dabei die Norm von $K$ bezüglich $k$. $\lambda$ wählen wir gleich 1 , falls $\chi=+1$, als Nichtnorm möglichst kleiner positiver Ordnung, falls $\chi=-1$ ist. $M_{0}$ ist dann die Maximalordnung von $K$.

$\Delta=w$. Ist $\chi=-1$, so ist $\lambda \sim p$. Nach (4.6) ist dieser Fall schon ausgeschaltet. Ist $\chi=+1$, so folgt aus (4.6) $\left(h_{0}, M_{0}\right)=A$, weil $M_{0}$ eine Determinante $\sim 1$ besitzt. Für beide Räume kommt nur $v=0$ vor.

$\Delta=1+\left[p^{2 g+1}\right](g<\omega)$. Es ist $M_{0}=A+A p^{-8}(1+V \bar{\Delta})$ (s. z. B. [2], S. 37). Für $h_{0}=\xi+\eta p^{-g}(1+V \bar{\Delta})$ berechnet man $\left(h_{0}, M_{0}\right)=\left(2 \xi+2 p^{-8} \eta\right) A+\left(2 p^{-g} \xi+\right.$ $\left.2 \eta p^{-2 g}(1-\Delta)\right) A$. Nach (4.6) ist $h_{0}$ sicher primitiv, also $\xi$ oder $\eta$ Einheit. Daher ist $\left(h_{0}, M_{0}\right)=\omega-g$. Es muß $v \leqq \omega-g$ sein.

Ist $\chi=+1$, so tritt $\nu=\omega-g$ auf. Beispiel: $h=h_{0}=1$.

Sei $\chi=-1$. Aus einem bekannten Satz der Verzweigungstheorie (s. [5], S.234, Cor.2) folgt: $K$ besitzt keine Nichtnormen in $1+\mathfrak{p}^{2(\omega-8)}$, wohl aber eine Nichtnorm $1+\gamma p^{2(\omega-g)-1}(\gamma \sim 1)$; die wir als $\lambda$ wählen. (4.4), (4.5) zeigen: Es muß $2 v \leqq \operatorname{ord}\left(\alpha_{1} \alpha_{2}\right) \leqq 2(\omega-g)-1$ sein, also $v \leqq \omega-g-1 . v=\omega-g-1$ kommt vor. Beispiel: $h=1+p^{\omega-g-1} f_{1}-\gamma p^{\omega-g} f_{2}$.

$\Delta \sim p$. Es ist $M_{0}=A+A \sqrt{\Delta}$ (s. z. B. [2], S.37). Ist $\chi=+1$, so kommt $v=\omega$ vor, z. B. für $h=h_{0}=1$. Sei $\chi=-1$. Wir können $\Delta=w=1+4 \varepsilon(\varepsilon \sim 1)$ wählen. $v=\omega$ kommt wieder vor, z. B. für $h=1+2 f_{1}-2 \varepsilon f_{2}$. 
$\operatorname{dim} \mathfrak{A}_{0}=3 . K$ sei die unverzweigte quadratische Erweiterung $k(\sqrt{w})$ von $k$, $N$ ihre Normform. Wir können $\mathfrak{I}_{0}$ identifizieren mit einer direkten Summe $k a \oplus K$ unter der Form

$$
M N(\xi a+y)= \begin{cases}\xi^{2} \Delta w+p N(y) & \text { falls } \Delta \sim 1 \\ \xi^{2} \Delta w+N(y) & \text { falls } \Delta \sim p .\end{cases}
$$

$M$ ist dann das Gitter $A a \oplus L$, wobei $L$ die Maximalordnung von $K$ bezeichne (s. [2], Satz 9.7).

Ist $\Delta \sim p$, so sieht man sofort, daß für jeden Vektor $h_{0}$ aus $M_{0}$, dessen Länge Einheit ist, $\left(h_{0}, M_{0}\right)=A$ ist. Es kommt nur $v=0$ vor.

$\Delta=1$. Es kommt $v=\omega$ vor. Beispiel: $h=a+2 f_{1}-2 \varepsilon f_{2}$. Dabei sei $\varepsilon$ die Einheit $\frac{1}{4}(w-1)$.

$\Delta=w$. Es kommt ebenfalls $v=\omega$ vor. Beispiel: $h=h_{0}=w^{-1} a$.

$\Delta=1+\left[p^{2 g+1}\right](g<\omega)$. Es ist $\Delta w=1+\eta p^{2 g+1}$ mit $\eta \sim 1$. Sei $h_{0}=\xi a+p^{t} z$ mit primitivem $z \in L$. Wir erhalten aus (4.4):

$$
M N\left(h_{0}\right)=1-\alpha_{1} \alpha_{2}=\xi^{2}\left(1+\eta p^{2 g+1}\right)+p^{2 t+1} M N(z) .
$$

Ist $t \neq g$, so muß ord $\left(\alpha_{1} \alpha_{2}\right) \leqq 2 g+1$, also $v \leqq g$ sein. Das liest man leicht aus (4.7) ab, unter Berücksichtigung der Tatsache, daß $\xi^{2}\left(1+\eta p^{2 g+1}\right)=1+\left[p^{\mu}\right]$ ist mit $\mu=2 g+1$ oder $\mu \leqq 2 g$ und gerade (s. [4], 63:5). Ist $t=g$, so muß $\left(h_{0}, M_{0}\right)$ $=2 A+\mathfrak{p}^{g+1}=\mathfrak{p}^{g+1}$ sein, also $v \leqq g+1$ ausfallen. $v=g+1$ kommt vor, wie das Beispiel $h=h_{0}=a+p^{g} z$ mit einem $z \in L$ der Norm $-\eta$ zeigt.

$\operatorname{dim} \mathfrak{A}_{0}=4$. Wir fassen $\mathfrak{A}_{0}$ als die nullteilerfreie Quaternionenalgebra über $k$ unter der Normform $N$ auf. $K:=k(\sqrt{w})$ läßt sich als Teilalgebra von $\mathfrak{N}_{0}$ auffassen und gibt Anlaß zu einer orthogonalen Zerlegung $\mathfrak{A}_{0}=K \oplus v K$ mit einem Element $v$ der Norm $p$. Es ist $M_{0}=L \oplus v L$, wobei $L$ wieder die Maximalordnung von $K$ bezeichne (s. [2], Satz 9.7). Ist $h_{0}=x+v y(x \in L, y \in L)$ Vektor mit $N\left(h_{0}\right)=N(x)+p N(y) \sim 1$, so muß $N(x) \sim 1$ sein. Daher ist $\left(h_{0}, M_{0}\right)$ $=A$. Es kommt nur $v=0$ vor.

\section{Literatur}

[1] Albert, A. A.: On Jordan algebras of linear transformations. Trans. Amer. Math. Soc. 69, 503- 527 (1950).

[2] EICHLER, M.: Quadratische Formen und orthogonale Gruppen. Grundl. d. Math. W. 63. Berlin-Göttingen-Heidelberg: Springer 1952, 1-220.

[3] KnebusCh, M.: Der Begriff der Ordnung einer Jordanalgebra. Abh. Math. Sem. Univ. Hamburg 28 (im Druck).

[4] O'Meara, O.T.: Introduction to quadratic forms. Grundl. d. Math. W. 117. BerlinGöttingen-Heidelberg: Springer 1963, 1-342.

[5] Serre, J.P.: Corps locaux. Act. Sci. Ind. 1296. Paris: Hermann 1962, 1-243.

[6] SIEgel, C.L.: Uber die analytische Theorie der quadratischen Formen. II. Ann. of Math. 36, 230-263 (1935).

[7] WrrT, E.: Theorie der quadratischen Formen in beliebigen Körpern. J. reine u. angew. Math. 176, 31- 44 (1936).

\section{Hamburg, Mathematisches Seminar der Universität}

(Eingegangen am 15. März 1965) 